\title{
Pain Relief and Imaging Improvement in Gammagraphy in Osteoid Osteoma after Etoricoxib Treatment in 20 Patients
}

\author{
José Carlos Minarro ${ }^{1 *}$, Carlos Alfonso-Bravo ${ }^{1}$, Alberto Izquierdo-Fernández ${ }^{1}$, María Teresa Urbano- \\ Luque $^{2}$ and Pedro Carpintero-Benítez ${ }^{1}$
}

${ }^{1}$ Department of Orthopedic Surgery, Hospital Reina Sofía, Córdoba, Spain

${ }^{2}$ Department of Animal Medicine and Surgery, University of Córdoba, Córdoba, Spain

Received: June 14, 2018; Published: June 27, 2018

*Corresponding author: José Carlos Minarro, Department of Orthopedic Surgery, Hospital Reina Sofía, Córdoba, Spain

Abstract

Purpose: Pain in Osteoid osteoma has always been treated with Non Steroid Anti-Inflammatory Drugs (NSAIDs) with satisfactory results. Recent researches reveal that pain is related to cyclooxigenase-2 (COX-2) secretion. The objective of this paper is to evaluate the pain response in patients with Osteoid osteoma after the administration of a selective COX-2 inhibitor (Etoricoxib).

Methods: A prospective study was made of consecutive patients diagnosed in our centre for Osteoid osteoma between January 2010 and December 2016. A total of 20 patients were enrolled (15 men and 5 women). Pain was collected in 3 stages: 1) treatment with NSAIDs 2) 1 month after treatment with Etoricoxib and 3) Final pain, 2 years after inclusion in the study. A Visual Analogue Scale (VAS) was used to determine the pain. Six months after starting treatment, possible gammagraphy changes due to treatment were assessed in patients who refused surgical treatment by radiofrequency (RFA).

Results: One month after treatment with Etoricoxib the nocturnal pain improved from $1.85 \pm 0.59$ to $0.93 \pm 0.41$, and the diurnal pain improved from $1.58 \pm 0.50$ to $0.40 \pm 0.30$ compared to when they were taking conventional NSAIDs ( $<<0.05)$. Gammagraphy was assessed in five of the patients finding no trazer uptake. Final VAS found no pain differences between those patients who underwent RFA and those who did not.

Conclusion: Treatment of Osteoid osteoma with Etoricoxib is more effective than conventional NSAIDs, and may be a better treatment for those patients who refuse a surgical treatment or if it is not indicated due to excessive morbidity.

Keywords: Pain; Osteoid osteoma; Gammagraphy; Etoricoxib; Treatment; Radiofrequency; Cyclooxigenase-2; COX-2

Abbreviations: NSAIDs: Non Steroid Anti-Inflammatory Drugs; COX-2: Cyclooxigenase-2; VAS: Visual Analogue Scale; RFA: Radio Frequency Ablation

\section{Introduction}

Osteoid osteoma is one of the most common benign bone tumour. Although it can affect any bone, it usually appears in long bones of young patient [1,2]. The clinical features are well-known as moderate-severe pain, typically worse at night and relieved by nonsteroid anti-inflammatory drugs (NSAIDs) [3].

As long-term intake of NSAIDs may lead to serious side-effects $[3,4]$, surgical ablation of the tumour is usually the treatment of choice $[5,6]$. However, medical treatment should be considered when percutaneous intervention is associated with significant morbidity [7]. The association between Osteoid osteoma and production of cyclooxigenase-2 (COX-2) has recently resulted in successful studies using a COX-2 inhibitor (Rofecoxib) for pain relief $[7,8]$.
Due to the recall in 2004 of Rofecoxib, the aim of this paper was to evaluate the effect of Etoricoxib (another COX-2 inhibitor) in pain caused by Osteoid osteoma and how the treatment may affect the evolution of the tumour in Gammagraphy.

\section{Materials and Methods}

All consecutive patients diagnosed in our centre of an Osteoid osteoma since January 2010 to December 2016 were prospectively enrolled. A total of 20 patients were included in the study (15 men and 5 women). Mean age was 16, 9 years old (range: 14-22). Tumors were located in tibia $(n=9)$, femur $(n=6)$, spine $(n=3)$, fibula $(n=1)$ and calcaneus $(n=1)$. All patients had previously been treated with conventional NSAIDs (Diclofenac, Ibuprofen, Ketorolac and 
Naproxen) selected by their general physician depending on tolerance to side-effects.

All patients received a daily single dose of Etoricoxib $90 \mathrm{mg}$. All prior NSAIDs or any analgesic therapies were discontinued. After explaining the procedure, all patients were offered surgery treatment by radiofrequency. 15 patients preferring this option received Etoricoxib treatment until surgery was performed. 5 patients preferring non-operative treatment received prescribed Etoricoxib daily during six months. After that, possible changes in gammagraphy due to treatment were evaluated.

Visual Analog Scale (VAS) was used to determine pain perception, where 0 represented no pain and 10 unbearable pain. Pain was indeed assessed in 3 stages:

a) Stage 1: Pain recalled by patients without receiving any treatment.

b) Stage 2: Pain with conventional NSAIDs.

c) Stage 3: Pain after 1 week of Etoricoxib treatment.

Differences between night-pain and day-pain were also assessed. Normality of the data distributions was checked by the Kolmogorov-Smirnov test. The results were expressed as mean \pm standard deviation. Student's T test and Wilcoxon's signed rank test were used to compare the results of pain during night and day in the 3 different stages. All statistical analyses were performed using SPSS statistic package v15.0 (SPSS Institute Inc. Headquarters, Chicago, IL, USA). The level of significance was set at $\mathrm{P}<0.05$.

\section{Results}

The main results are shown in Tables $1 \& 2$. Pain perception was worse when patients had no treatment and it decreased on administration of conventional NSAIDs. However, all patients referred a remarkably improvement, or even absence, of pain in the first week after taking the selective COX-2 compared to when they were taking conventional NSAIDs.

Table 1: Patient pain perception measured on Visual Analogue Scale.

\begin{tabular}{|c|c|c|c|}
\hline Pain & No Treatment & Conventional NSAIDs* & Etoricoxib \\
\hline Nocturnal & $6.9 \pm 0.68$ & $1.85 \pm 0.59$ & $0.93 \pm 0.41$ \\
\hline Diurnal & $4 \pm 0.71$ & $1.58 \pm 0.50$ & $0.40 \pm 0.30$ \\
\hline
\end{tabular}

Data is shown as Mean \pm SD. $(\mathrm{P}<0.001)$.

*NSAIDs: non-steroidal anti-inflammatory drugs.

Table 2: Final pain after 2 years of follow-up.

\begin{tabular}{|c|c|c|}
\hline Patients & RFA* $^{*}(\mathbf{n}=\mathbf{1 5})$ & Etoricoxib $^{* *}(\mathbf{n}=\mathbf{5})$ \\
\hline Final Pain & $0.2 \pm 0.25$ & $0.50 \pm 0.35$ \\
\hline
\end{tabular}

Data is shown as Mean \pm SD. $(\mathrm{P}>0.05)$.

*RFA: Radiofrequency Ablation.

**Etoricoxib was used daily during 6 months, after that, only used on demand.

In five of the patients (whom refused surgical treatment by radiofrequency ablation) the gammagraphy was normal after six months (Figure 1), although a more attenuated pain still persisted. Neither side effects nor intolerances were reported.

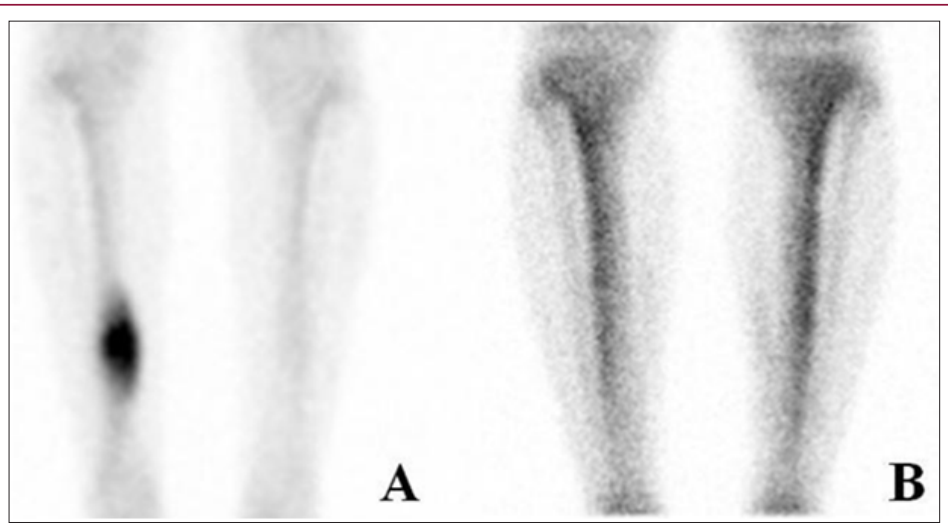

Figure 1: Here are shown the images of one of the patients that refused surgical treatment by radiofrequency: before taking Etoricoxib (A) and after six months of treatment (B). We can observe how the tracer uptake of the Osteoid osteoma in A completely disappears in B.

\section{Discussion}

Osteoid osteoma is one of the most common (11-14\%) [1] benign bone tumor firstly described by Jaffe in 1935 [9]. This osteogenic tumor appears in younger patients usually under the age of 30 , although $50 \%$ of patients aged between 10 and 20 years old [2]. Osteoid osteoma is 1.6-4 times more prevalent in males [1]. The most common location are the metaphysis of long bones (femur and tibia), although it can involve any bone [10]. Clinical features of this tumor are mild and intermittent pain that increases in intensity over time and becomes severe at night [1]. Swelling, tenderness or erythema may appear in subcutaneous locations [11] and if continues over time muscular atrophy might occur [12]. Diagnosis of an Osteoid osteoma is clinical but it requires complementary exam such as plain radiographs and computed tomography to give an accurate diagnosis and exclude other diseases or tumors $[1,10]$. Bone gammagraphy is also useful for diagnosis as radiotracer uptake can be easily found in the region of the nidus $[13,14]$.

As a benign tumor, the natural evolution of Osteoid osteoma is spontaneous resolution after an average of 2 to 5 years $[3,15,16]$. Non-operative treatment with NSAIDs has reported relief of the 
symptoms as well as regression of the nidus in about 30-40 months [3]. However, prolonged NSAIDs intake has potentially serious side-effects $[3,4]$. Operative treatment using percutaneous CT guidance such as radiofrequency ablation (RFA) is almost always successful in resolving the pain $[5,6]$. However, general anaesthesia and surgery always involves a small morbidity, not acceptable for some patients [17].

The mechanism of how pain appears in Osteoid osteoma and its relief with NSAIDs is still controversial. Many authors have found a relationship with COX-2 $[7,8,18]$. Osteoid osteoma consists of a nidus that is surrounded by sclerotic bone, which density varies with time from the onset of the injury [19]. Increased local concentrations of prostaglandins (PGs) have been described in the nidus $[1,18,20,21]$. Prostaglandins are related with vasodilatation and pain [3,7]. Increased PGs values are reversible after osteoma removal [21]. Apparently, the nidus displays a strong diffuse staining for COX-2, which is a key enzyme in the production of PGs [18]. Thus, selective COX-2 inhibition may enable a successful control of the symptoms $[7,8]$.

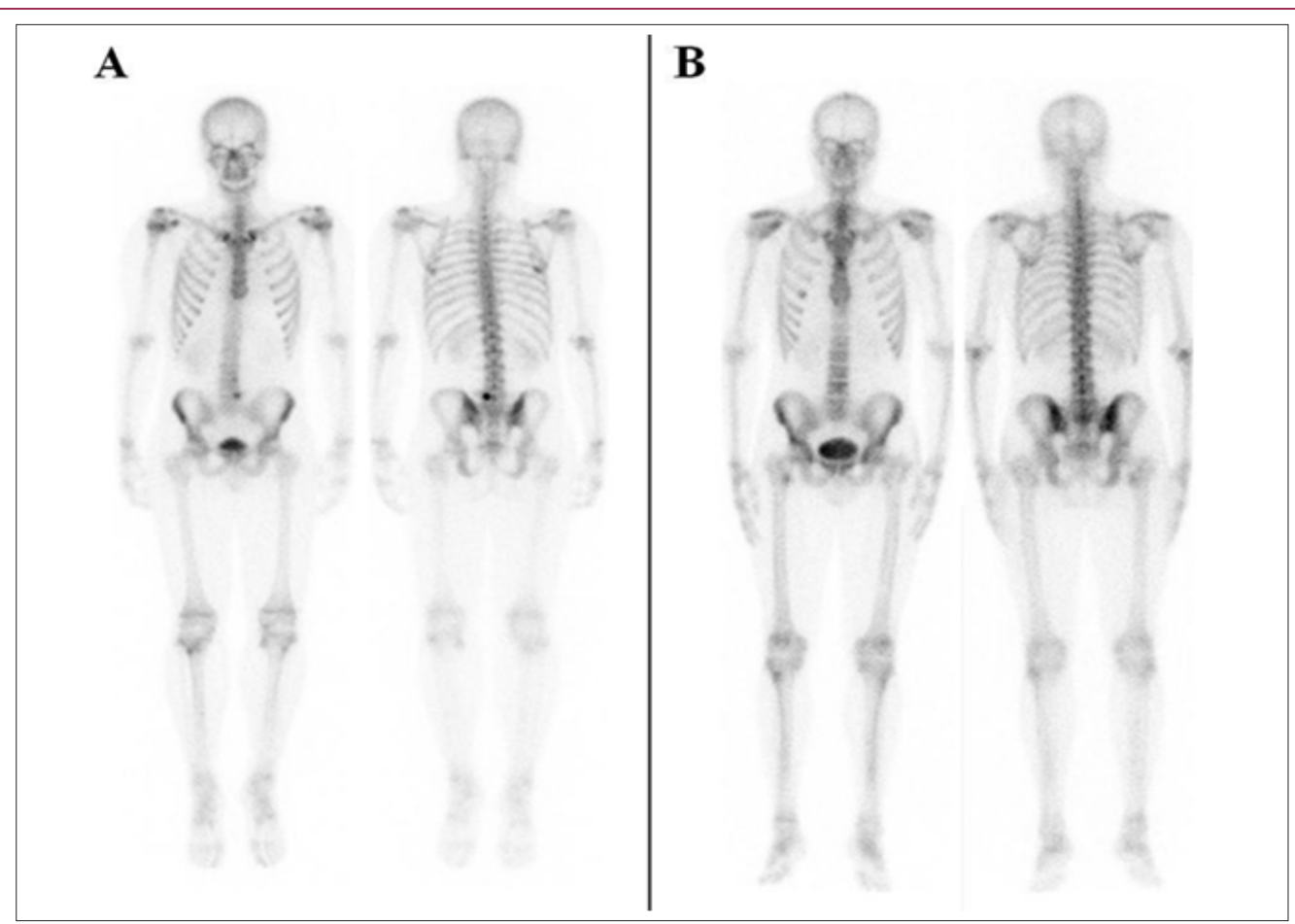

Figure 2: Here is shown the gammagraphy of one of the patients who had an Osteoid osteoma in L5. We can observe how the tracer uptake and even the antialgic scoliotic attitude seen in A (before taking Etoricoxib) completely disappears in B (after six months of treatment).

In our study we use this relationship between COX-2 and the Osteoid osteoma. A selective COX-2 inhibitor (Etoricoxib) was used in 20 patients. A significative difference in pain relieve was found when compare to NSAIDs, the gold standard medical treatment $[1,3,22]$. A month after starting the treatment VAS was evaluated, the night pain improved from $1.85 \pm 0.59$ to $0.93 \pm 0.41$, and the day pain improved from $1.58 \pm 0.50$ to $0.40 \pm 0.30$. Thus, our study showed that Etoricoxib relieves pain much better than NSAIDs therapy $(\mathrm{p}<0.05)$.

All patients (20) were offered radiofrequency ablation (RFA) as a definitive treatment. Five patients (25\%) decline operative treatment and decided to continue with Etoricoxib. A control gammagraphy was performed after six months, showing an absence of tracer intake in all 5 patients (Figure 2). Pain was minimal and bearable with sporadic analgesia on demand. Etoricoxib effect in pain relief is equivalent to Rofecoxib $[7,8]$ because both are drugs from the same group. Although these results should be viewed with caution due to the small number of patients involved, our study shows how selective COX-2 inhibitors could be useful for the resolution of Os- teoid osteoma. The strengths of the study are the long follow-up of both pain and gammagraphy, never described before.

\section{Conclusion}

Selective COX-2 inhibitor (Etoricoxib) has demonstrate to be an effective treatment for pain due to Osteoid osteoma. Moreover, a long-term use (at least 6 months) would wipe out uptake in gammagraphy, so it may be considered as a better treatment than conventional NSAIDs. Future research could be aimed at studying whether selective COX-2 inhibitor may actually be a definitive treatment for Osteoid osteoma.

\section{References}

1. Boscainos PJ, Cousins GR, Kulshreshtha R (2013) Osteoid osteoma. Orthopedics 36: 792-800.

2. Frassica F, Waltrip R, Sponseller P (1996) Clinicopathologic features and treatment of osteoid osteoma and osteoblastoma in children and adolescents. Orthop Clin North Am 27(3): 559-574.

3. Kneis J, Simon M (1992) Medical management compared with operative treatment of osteoid-osteoma. J Bone Jt Surg 74(2): 179-185. 
4. Atchison J, Herndon C, Rusie E (2013) NSAIDs for musculoskeletal pain management: Current perspectives and novel strategies to improve safety. J Manag Care Pharm 19: 3-19.

5. Campanacci M, Ruggieri P, Basbarrini A (1999) Osteoid osteoma: Direct visual identification and intralesional excision of the nidus with minimal removal of bone. J Bone Jt Surg Br 81(5): 814-820.

6. Gebauer B, Collettini F, Bruger C (2013) Radiofrequency ablation of osteoid osteomas: Analgesia and patient satisfaction in long-term follow-up. RoFo 184(10): 959-966.

7. Bottner F, Roedl R, Wortler K (2001) Cyclooxygenase-2 inhibitor for pain management in osteoid osteoma. Clin Orthop Relat Res 393: 258-263.

8. Carpintero-Benitez P, Aguirre MA, Serrano JA, Lluch M (2004) Effect of rofecoxib on pain caused by osteoid osteoma. Orthopedics 27(11): 1188-1191.

9. Jaffe H (1935) Osteoid-osteoma: A benign osteoblastic tumor composed of osteoid and atypical bone. Arch Surg 31(5): 709-728.

10. Jordan RW, Koç T, Chapman AWP, Taylor HP (2015) Osteoid osteoma of the foot and ankle-A systematic review. Foot Ankle Surg 21(4): 228-234.

11. Gitelis S, Schajowicz F (1989) Osteoid osteoma and osteoblastoma. Orthop Clin North Am 20: 313-325.

12. Sim F, Dahlin D, Stauffer R, Laws EJ (1977) Primary bone tumors simulating lumbar disc syndrome. Spine 2: 65-74.

(C) This work is licensed under Creative

Submission Link: https://biomedres.us/submit-manuscript.php
13. Smith F, Gilday D (1980) Scintigraphic appearances of osteoid osteoma. Radiology 137: 191-195.

14. Helms C, Hattner R, Vogler JI (1984) Osteoid osteoma: radionuclide diagnosis. Radiology 151(3): 779-784.

15. Leicester A, Trantalis J (2001) Osteoid osteoma in a young child: Successful non-operative management. ANZ J Surg 71(8): 491-493.

16. Spouge A, Thain L (1999) Osteoid osteoma: MR imaging of two untreated lesions. Clin Imaging 23(4): 254-258.

17. Mylona S, Patsoura S, Galani P (2010) Osteoid osteomas in common and in technically challenging locations treated with computed tomographyguided percutaneous radiofrequency ablation. Skelet Radiol 39(5): 443449.

18. Mungo D, Zhang X, O'Keefe R (2002) COX-1 and COX-2 expression in osteoid osteomas. J Orthop Res 2(1): 159-162.

19. Golding J (1954) The natural history of osteoid osteoma; with a report of twenty cases. J Bone Jt Surg Br 36: 218-229.

20. Makley J, Dunn M (1982) Prostaglandin synthesis by osteoid osteoma. Lancet 2(8288): 42.

21. Greco F, Tamburrelli F, Ciabattoni G (1991) Prostaglandins in osteoid osteoma. Int Orthop 15(1): 35-37.

22. Munk PL, Huk ME (2003) Medical management of osteoid osteoma. Can J Surg 46(1): 60-61.

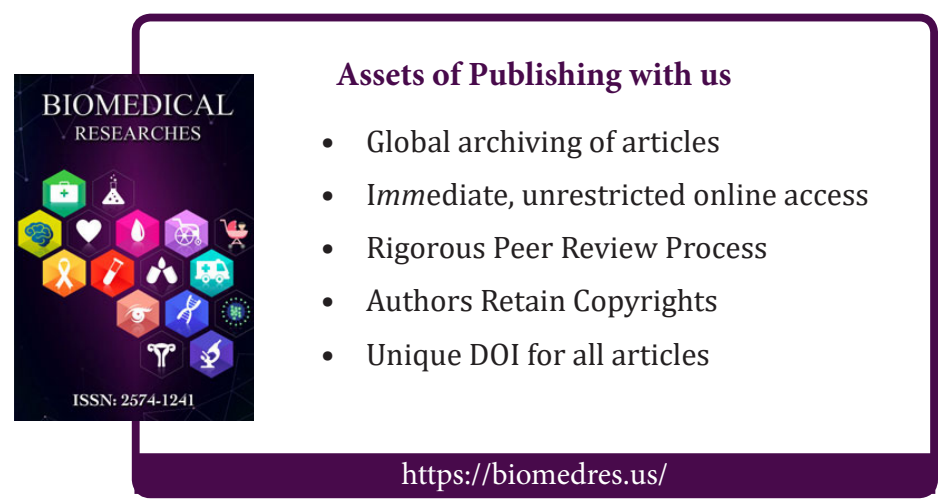

